\title{
Validación de un instrumento para evaluar calidad de vida asociada a hipertrofia mamaria en mujeres chilenas
}

\author{
GÜNTHER MANGELSDORFF G. ${ }^{1,2}$, FERNANDA DEICHLER V., \\ M. ANGÉLICA GARCÍA-HUIDOBRO D. ${ }^{4}$, ESTEFANÍA ENRÍQUEZ CH. ${ }^{5, a}$, \\ GUILLERMO ISRAEL V. ${ }^{1}$, M. TERESA PESQUEIRA B. ${ }^{1}$
}

\section{Validation of a Spanish version of the breast related symptoms questionnaire for breast hypertrophy}

Background: Breast hypertrophy has been associated with a significant impact on quality of life. Aim: To validate a specific scale to evaluate quality of life of Chilean women with symptomatic breast hypertrophy. Material and Methods: We included 80 women, 40 patients ( 20 undergoing reduction mammoplasty) and 40 controls. The Breast Related Symptoms (BRS) questionnaire in Spanish was used. The results are expressed as a score ranging from 0 to 100, with higher values representing better specific quality of life. Internal consistency, reliability and validity of the test were evaluated. The results of patients and controls and the scores of patients undergoing reduction mammoplasty, before and after surgery, were compared. A receiver operating characteristic (ROC) curve was constructed to define the score cutoff point defining symptomatic breast hypertrophy. Results: The Cronbach's alpha was 0.96, linear weighed kappa test-retest was 0.87 [95\% confidence intervals (CI) 0.31-1] and inter-observer variability was 0.79 [95\% CI 0.71-0.87]. Participants with breast hypertrophy had a lower specific quality of life than the control group (25 (2-71) and 91 (25-100) respectively, $p<0.01)$. In patients undergoing surgery, the preoperative score was lower than the postoperative figures (37 (12-71) and 96 [94-100] respectively, $p<0.01)$. The area under the ROC curve was $0.97(p<0,001)$ and the cutoff point for symptomatic breast hypertrophy was a score below 72.1. Conclusions: The Spanish version of the BRS questionnaire is a valid and reliable instrument to measure specific quality of life in Chilean women with mammary hypertrophy.

(Rev Med Chile 2013; 141: 1143-1149).

Key words: Breast; Mammaplasty; Quality of life; Questionnaires.

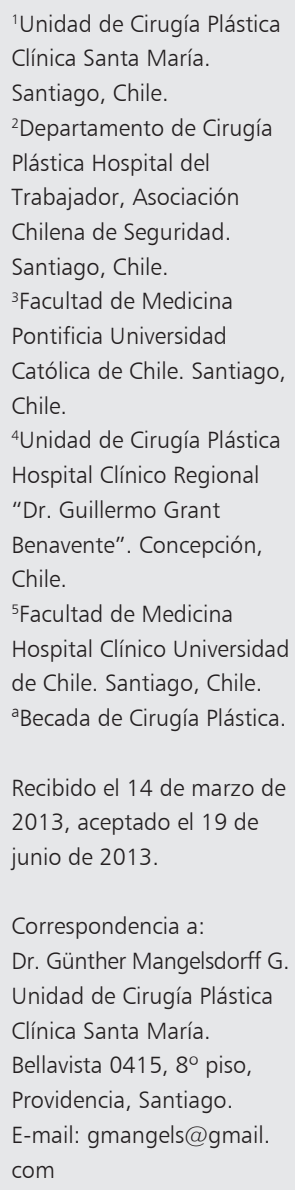

L a calidad de vida relacionada a salud (CVRS) es la percepción subjetiva, influida por el estado de salud actual, de la capacidad para realizar aquellas actividades importantes para el individuo $^{1,2}$. Incluye dimensiones como el funcionamiento físico, emocional, cognitivo, social, percepción de salud general y síntomas específicos de enfermedad ${ }^{3,4}$. La evaluación de CVRS mide el impacto de la enfermedad y/o el tratamiento en el bienestar de los pacientes, aporta una medida del resultado incorporando la percepción del paciente y, como evento no observable, su medición se considera subjetiva ${ }^{3-5}$. La evaluación de CVRS puede ser realizada a través de: a) instrumentos genéricos (ej. SF-36, EuroQOL); b) instrumentos específicos: enfermedades particulares (asma, cáncer, etc.), grupos de pacientes, o c) áreas o dimensiones de funcionamiento, por ejemplo, 
dolor ${ }^{6-9}$. Se han creado diversos instrumentos que tienen como objetivo resumir en un índice las diferentes variables que los pacientes consideran afectan su CVRS ${ }^{3}$.

La hipertrofia mamaria se refiere al volumen mamario excesivo y este puede afectar la CVRS. Se clasifica, según el volumen que será necesario resecar en: leve (menos de 200 (500) g), moderada (entre 200 y $500 \mathrm{~g}$ ), mayor (más de $500 \mathrm{~g})^{10}$. Las pacientes portadoras de hipertrofia mamaria refieren diversos síntomas y alteraciones en sus actividades cotidianas en relación al tamaño y peso de sus mamas. Pueden presentar cefalea, dolor cervical, de hombros, dorsal, lumbar, peso mamario, intertrigo persistente bajo las mamas, surcos y marcas por el sostén; así como dificultades para encontrar ropa adecuada y problemas para realizar actividad física ${ }^{11,12}$. Además, trastornos psíquicos originados por la fobia dismórfica, que afectan la autoestima y las relaciones interpersonales ${ }^{13-15}$.

La medición de la gravedad de estos síntomas $\mathrm{y}$ de las alteraciones en actividades cotidianas, permitiría obtener información del grado de compromiso de la calidad de vida en estas pacientes, con ello orientar la indicación de cirugía y documentar los resultados con diferentes alternativas terapéuticas.

Numerosos estudios han tratado de cuantificar la repercusión en la calidad de vida, algunos utilizando escalas que evalúan aspectos más generales, como por ejemplo, the health utilities index mark2, mark 3 y Short form 36 (SF-36); y otras mediante instrumentos que son considerados específicos para hipertrofia mamaria: the multidimensional body self relations questionnaire appearance scales, Breast-Q y Breast Related Symptoms questionnaire (BRS) ${ }^{16-20}$. Actualmente, no contamos con instrumentos específicos de este tipo, que hayan sido validados en español, ni en mujeres chilenas portadoras de hipertrofia mamaria.

Nuestro objetivo es validar una escala específica para evaluar la calidad de vida asociada a hipertrofia mamaria en mujeres chilenas.

\section{Pacientes y Método}

\section{Diseño y pacientes}

Estudio de corte transversal, participaron ochenta mujeres (cuarenta pacientes y cuarenta controles). Los criterios de inclusión de pacien- tes fueron: mayores de 15 años, portadoras de hipertrofia mamaria sintomática, candidatas a reducción mamaria y consentimiento voluntario para completar el cuestionario. Fueron excluidas las pacientes con antecedente de cirugía mamaria, enfermedad reumatológica, patología de columna $\mathrm{u}$ obesidad mórbida. Las pacientes se obtuvieron de mujeres que consultaron por hipertrofia mamaria sintomática entre el 2009 y 2010, que cumplieron los criterios de inclusión. De estas mujeres, se seleccionaron al azar veinte pacientes que fueron operadas (reducción mamaria) y veinte pacientes que no fueron operadas durante el período mencionado. Los controles (cuarenta pacientes), fueron mujeres sin hipertrofia mamaria sintomática seleccionadas al azar entre mujeres que consultaron por cirugía estética no mamaria y que aceptaron participar en el estudio, excluyendo aquellas que presentaran alguno de los criterios arriba señalados.

Las variables registradas incluyeron edad, peso y talla. Se calculó en índice de masa corporal (IMC, $\mathrm{kg} / \mathrm{m}^{2}$ ). En el grupo pacientes, se incluyó además la distancia entre escotadura esternal y complejo areola pezón $(\mathrm{cm})$, y en aquellas sometidas a reducción mamaria, se registró el peso del tejido resecado total (g).

\section{Cuestionario}

El cuestionario, que evalúa aspectos de la calidad de vida relacionados a la hipertrofia mamaria denominado BRS (Breast Related Symptoms), fue desarrollado por Kerrigan y cols. ${ }^{19}$, para esta patología. Es un instrumento simple y conciso, que mediante trece preguntas, evalúa la presencia y gravedad de los síntomas y alteraciones atribuibles al tamaño de las mamas. Cada ítem tiene una escala de categorías tipo Likert, que asigna puntajes de 1 a 5 ; donde 1 corresponde a un síntoma o alteración siempre presente y 5 si nunca lo está. Se calcula un puntaje total sumando los puntos de cada ítem y mediante una transformación lineal se lleva a escala de 0 a 100. A mayor puntaje mejor calidad de vida, es decir, los síntomas y alteraciones evaluadas son menos frecuentes o están ausentes. Su validez de contenido y de fachada fue establecida en su desarrollo original por participación de un panel de expertos y focus groups de pacientes con hipertrofia mamaria. Si bien su consistencia interna no ha sido adecuadamente documentada en estudios disponibles, ha conseguido validación 
parcial a través de una alta fiabilidad test-retest. Luego de obtener la autorización expresa de los autores, se realizó la traducción y retrotraducción del cuestionario al español (Tabla 1). La aplicación del cuestionario fue realizada por encuestador entrenado.

Se calculó un tamaño muestral mínimo de 40 pacientes por grupo, para pesquisar una diferencia de 10 puntos con un alfa de 0,05 y una potencia de $80 \%$, considerando una desviación estándar de 16 , obtenida de estudios previos.

El mismo cuestionario fue aplicado a controles y a pacientes antes y después de la cirugía.

\section{Método de validación}

En el proceso de validación se evaluó la consistencia interna, confiabilidad y validez de la escala BRS en español.

La consistencia interna estima la fiabilidad de pruebas cuando se utilizan conjuntos de ítems, que se espera midan el mismo atributo o campo de contenido. Se calculó mediante el coeficiente alfa de Cronbach, que ofrece una estimación única de fiabilidad y que depende de la longitud de la prueba y de la covarianza de sus ítems. Se calculó el alfa de Cronbach de la prueba completa y el coeficiente obtenido al excluir cada ítem; así se puede determinar si alguno de ellos no logra medir en forma adecuada el mismo atributo que el resto de la prueba. Alcanza valores entre 0 y 1 , cuanto más se aproxime a 1 mayor es la fiabilidad de la escala ${ }^{21-24}$.

La confiabilidad de la escala, es decir, el grado de acuerdo o concordancia entre las mediciones realizadas en momentos diferentes o por observadores distintos, se evaluó mediante la confiabilidad test-retest y la confiabilidad interobservador ${ }^{23}$. Así, veinte pacientes fueron encuestadas y reencuestadas dos semanas después de la primera evaluación, sin mediar cirugía, para evaluar la estabilidad temporal del instrumento. A diez de ellas la encuesta se las realizó el mismo evaluador (confiabilidad test-retest) y a las diez restantes la entrevista se las hizo un segundo evaluador ciego a los resultados de la primera evaluación (confiabilidad interobservador).

Finalmente, se analizó la validez de la escala, es decir, el grado en que mide aquello que realmente pretende medir, o su utilidad para el propósito que ha sido diseñada ${ }^{23}$. La validez de constructo fue evaluada de dos formas: 1) comparando los
Tabla 1. Cuestionario de síntomas relacionados a hipertrofia mamaria (BRS, Breast Related Symptoms)

\begin{tabular}{|c|c|}
\hline Ítem & Pregunta \\
\hline 1 & $\begin{array}{l}\text { El tamaño de mis mamas causa dolor en la parte } \\
\text { superior de la espalda }\end{array}$ \\
\hline 2 & $\begin{array}{l}\text { Por el tamaño de mis mamas tengo dificultad para } \\
\text { encontrar sostén y ropa }\end{array}$ \\
\hline 3 & $\begin{array}{l}\text { Por el tamaño de mis mamas tengo dolores de } \\
\text { cabeza }\end{array}$ \\
\hline 4 & Tengo dolor mamario \\
\hline 5 & $\begin{array}{l}\text { El tamaño de mis mamas causa dolor en la parte } \\
\text { inferior de la espalda }\end{array}$ \\
\hline 6 & Presento irritación o picazón bajo mis mamas \\
\hline 7 & Tengo marcas dolorosas por los tirantes del sostén \\
\hline 8 & $\begin{array}{l}\text { El tamaño de mis mamas me hace difícil participar } \\
\text { en deportes }\end{array}$ \\
\hline 9 & El tamaño de mis mamas causa dolor en el cuello \\
\hline 10 & $\begin{array}{l}\text { El tamaño de mis mamas causa dolor en los } \\
\text { hombros }\end{array}$ \\
\hline 11 & Me cuesta correr por el tamaño de mis mamas \\
\hline 12 & $\begin{array}{l}\text { Por el tamaño de mis mamas tengo dolor en mis } \\
\text { manos o se sienten adormecidas }\end{array}$ \\
\hline 13 & El tamaño de mis mamas causa dolor en los brazos \\
\hline
\end{tabular}

Versión en español del cuestionario de síntomas relacionados a la hipertrofia mamaria. Contiene trece preguntas que evalúan síntomas atribuibles al tamaño de las mamas. A cada ítem se asigna puntaje de 1 a 5 (1 corresponde a un síntoma siempre presente, y 5 nunca presente). El puntaje total se obtiene sumando los puntos y mediante una transformación lineal se lleva a escala de 0 a 100, donde mayores puntajesindican mejor calidad de vida.

resultados de la escala BRS obtenidos en pacientes portadoras de hipertrofia mamaria versus controles y 2) evaluando la capacidad de respuesta de la escala a los cambios, comparando los puntajes de escala BRS en 20 pacientes antes y después de ser sometidas a cirugía de reducción mamaria. El intervalo de tiempo entre la cirugía y la encuesta postoperatoria fue de seis meses.

\section{Análisis estadístico}

Las variables categóricas se expresan en frecuencia y porcentaje. Las variables numéricas se expresan en media \pm desviación estándar (DS) o en mediana (mínimo-máximo).

La comparación de proporciones se evaluó con 
Calidad de vida en mujeres con hipertrofia mamaria - G. Mangelsdorff et al

test $\chi^{2}$ o test exacto de Fisher. Las diferencias de variables numéricas fueron estudiadas con test de rangos pareados de Wilcoxon o U de Mann Whitney, dependiendo si las comparaciones fueron pareadas o independientes. Para comparaciones múltiples se utilizó Kruskal-Wallis con corrección de Bonferroni. La estimación de consistencia interna se midió mediante el alfa de Cronbach. La concordancia test-retest e interobservador se calculó mediante el estadístico kappa con ponderación lineal, por tratarse de categorías ordinales, ya que toma en cuenta la importancia relativa de los desacuerdos observados. También se calculó la correlación test-retest e interobservador utilizando el coeficiente rho de Spearman. El análisis multivariado se realizó mediante regresión lineal múltiple. Con los puntajes obtenidos en la escala, se confeccionó la curva ROC para determinar área bajo la curva, como indicador de la exactitud global de la prueba, y para definir el punto de corte con mayor sensibilidad y especificidad para predecir hipertrofia mamaria sintomática con alteración en la calidad de vida. Se utilizó el programa SPSS $16.0 .2^{\circledR}$.

\section{Resultados}

Las características clínicas de las pacientes y controles se muestran en la Tabla 2. No se observaron diferencias de edad, peso, talla o IMC entre ambos grupos. En pacientes, la mediana de distancia entre escotadura esternal y complejo areola-pezón fue $28 \mathrm{~cm}(25-38 \mathrm{~cm})$.

Todas las pacientes refirieron alteraciones en al menos 3 de los 13 ítems evaluados, mientras que $92 \%$ lo hizo en al menos 7 ítems y $25 \%$ presentó alteraciones en todos los ítems de la escala. Las alteraciones más frecuentes en las pacientes fueron dolor cervical, dorsal, de hombros, dificultades para correr, hacer deportes, para vestir, y marcas dolorosas por tirantes del sostén. En sujetos controles $23 \%$ no refirió ningún síntoma $(\mathrm{p}=0,002)$.

\section{Consistencia interna}

Se observó una alta consistencia interna, obteniéndose un alfa de Cronbach de 0,96 para la prueba completa, lo que indica una elevada fiabilidad de la escala. La Tabla 3 muestra el alfa de Cronbach si se excluyera cada uno de los ítems. Se observa que la exclusión de cada ítem no mejora significativamente el coeficiente, lo que señala que todos los ítems se encuentran intercorrelacionados midiendo el mismo atributo.

\section{Confiabilidad}

Tanto la estabilidad temporal como la confiabilidad interobservador fueron adecuadas. Se obtuvo un estadístico kappa ponderado lineal de 0,87 (IC 95\% 0,31-1) para la concordancia test-retest y de 0,79 (IC 95\% 0,71-0,87) para la concordancia interobservador. Del mismo modo, la correlación de Spearman test-retest fue de 0,97 $(\mathrm{p}<0,001)$ y

Tabla 2. Características de ambos grupos

\begin{tabular}{|lccc|}
\hline & Pacientes & Controles & Valor $\mathbf{p}$ \\
$\mathrm{n}$ & 40 & 40 & - \\
Edad (años) & $33 \pm 13$ & $33 \pm 11$ & 0,99 \\
Peso $(\mathrm{kg})$ & $66 \pm 10$ & $63 \pm 10$ & 0,38 \\
Talla $(\mathrm{m})$ & $1,60 \pm 0,6$ & $1,62 \pm 0,8$ & 0,31 \\
IMC $\left(\mathrm{kg} / \mathrm{m}^{2}\right)$ & $26 \pm 4$ & $25 \pm 4$ & 0,18 \\
\hline
\end{tabular}

Valores se expresan como media \pm desv. estándar. Valor $p$ según $U$ de Mann Whitney.

Tabla 3. Coeficiente Alfa de Cronbach

\begin{tabular}{|lc|}
\hline ítem & $\begin{array}{c}\text { Alfa de } \\
\text { Cronbach }\end{array}$ \\
\hline 1. Dolor en la parte superior de la espalda & 0,954 \\
\hline 2. Dificultad para encontrar ropa y sostén & 0,954 \\
\hline 3. Dolor de cabeza & 0,958 \\
\hline 4. Dolor mamario & 0,961 \\
\hline 5. Dolor en la parte inferior de la espalda & 0,955 \\
\hline 6. Irritación o picazón bajo las mamas & 0,956 \\
\hline 7. Marcas dolorosas por los tirantes del sostén & 0,953 \\
\hline 8. Dificultad para participar en deportes & 0,954 \\
\hline 9. Dolor de cuello & 0,952 \\
\hline 10. Dolor de hombros & 0,953 \\
\hline 11. Dificultad para correr & 0,954 \\
\hline 12. Dolor o adormecimiento de manos & 0,960 \\
\hline 13. Dolor de brazos & 0,959 \\
\hline
\end{tabular}

Coeficiente alfa de Cronbach obtenido al excluir cada ítem. La exclusión de cada ítem no mejora significativamente el coeficiente, indicando que todos los ítems se encuentran intercorrelacionados midiendo el mismo atributo. 


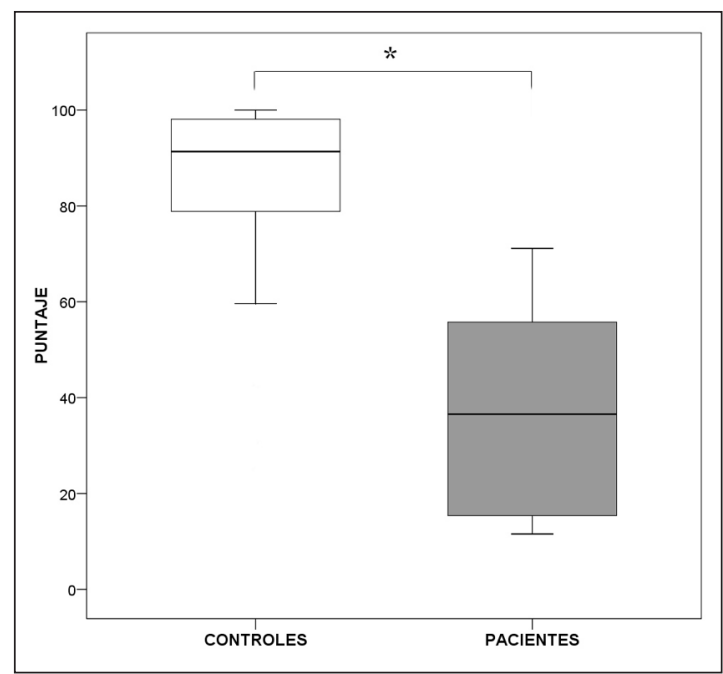

Figura 1. Puntajes escala de síntomas relacionados a hipertrofia mamaria (BRS, Breast Related Symptoms). Gráfico de cajas con puntajes de escala BRS obtenidos en controles (cajas blancas) y pacientes (cajas grises). * $p<0,001$ (Mann Whitney).

la interobservador de $0,94(\mathrm{p}<0,001)$.

\section{Validez}

El puntaje total de la escala en pacientes fue menor que la registrada en controles (Figura 1), mediana 25 (2-71) vs 91 (25-100) respectivamente ( $\mathrm{p}<0,001$, Mann Whitney). Así las pacientes presentan un mayor número de alteraciones y/o con mayor frecuencia que las mujeres sanas. Esta diferencia, se observó tanto para el puntaje final, como en cada uno de los ítems por separado, y se mantuvo al ajustar por las variables biodemográficas ( $p<0,001$ modelo de regresión lineal múltiple). En pacientes portadoras de hipertrofia mamaria, el peso del tejido mamario resecado en la mamoplastía de reducción tuvo una mediana de 892 gramos (508-2.070 gramos). El puntaje de la escala después de la cirugía fue mayor que el obtenido antes de ésta (Figura 2), mediana 96 (94-100) vs 37 (12-71) respectivamente $(\mathrm{p}<0,001$, Wilcoxon). La diferencia de puntaje (post-pre) tuvo una mediana de 60 puntos (25-87), diferencia significativa, tanto para el puntaje final, como para cada ítem por separado.

Se construyó una curva ROC, que posee un área bajo la curva de $0,97(\mathrm{p}<0,001)$ reflejando una alta exactitud global de la prueba. Se determinó un puntaje de 72,12 puntos como punto de

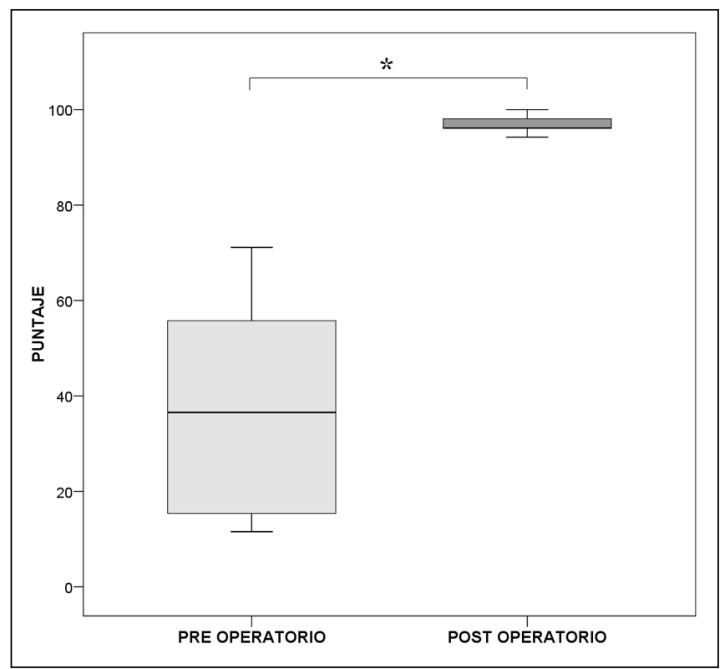

Figura 2. Puntajes escala de síntomas relacionados a hipertrofia mamaria (BRS, Breast Related Symptoms). Gráfico de cajas con puntajes de escala BRS obtenidos en pacientes portadoras de hipertrofia mamaria. Se compara preoperatorio (gris claro) y postoperatorio (gris oscuro). ${ }^{*} p<0,001$ (Wilcoxon).

corte, con una sensibilidad de $100 \%$ y especificidad de $85 \%$, para asociación con hipertrofia mamaria sintomática con alteración en la calidad de vida.

\section{Discusión}

El presente estudio muestra que la versión en español del cuestionario de síntomas relacionados a la hipertrofia mamaria (BRS) es un instrumento confiable y válido para medición de calidad de vida específica asociada a este problema de salud, en mujeres chilenas. Posee altos niveles de consistencia interna, estabilidad temporal y concordancia inter observador. Permite diferenciar con gran precisión a mujeres portadoras de hipertrofia mamaria sintomática con alteración en la calidad de vida, de aquellas que no sufren este problema. Además, es capaz de detectar cambios en la calidad de vida específica, tras el tratamiento quirúrgico.

Los síntomas más frecuentemente relacionados a la hipertrofia mamaria fueron dolor cervical, dorsal, de hombros; surcos dolorosos en hombros por el sostén, dificultad para correr, hacer deportes y para encontrar ropa adecuada. Esto es concordante con diferentes series publicadas en la literatura ${ }^{21-29}$. Las diferencias observadas entre 
mujeres del grupo control y pacientes portadoras de hipertrofia mamaria, tanto en la frecuencia de sus alteraciones, como en el puntaje obtenido por la escala BRS en español, permiten objetivar el impacto físico y afección de calidad de vida de estas pacientes.

La cirugía reductiva mamaria es uno de los procedimientos más comúnmente realizados por los cirujanos plásticos. Según datos aportados por la Sociedad Americana de Cirujanos plásticos, sólo en el año 2006, se realizaron alrededor de 100.000 cirugías de reducción mamaria en Estados Unidos de Norteamérica ${ }^{30}$. Sus objetivos pueden resumirse en: mejorar los síntomas asociados al tamaño mamario, reducir el volumen mamario conservando un complejo areola-pezón (CAP) irrigado e inervado, reposicionar el CAP, mejorar de manera estable la forma mamaria y minimizar las cicatrices ${ }^{30,31}$. Existe consenso en que la mamoplastía reductiva es un tratamiento definitivo y efectivo para la hipertrofia mamaria, aliviando la mayor parte de los síntomas asociados a esta patología $11,12,16-18,27,28,32-37$. Métodos conservadores como terapia física, baja de peso, uso de sostén especial y tratamiento hormonal (hipertrofia virginal) no han sido efectivos en brindar alivio permanente de los síntomas ${ }^{17,18,34}$. Los resultados obtenidos luego de una mamoplastía de reducción son habitualmente evaluados mediante análisis fotográfico y preguntas no estandarizadas sobre la opinión del paciente al respecto, utilizando generalmente un cuestionario telefónico sin describir ni utilizar escalas correctamente validadas para la medición de calidad de vida o sintomatología especifica en estas pacientes ${ }^{25,36}$. Mediante el uso de una escala simple y fiable, en nuestro estudio, observamos que las pacientes sometidas a cirugía de reducción mamaria tuvieron una mejoría significativa de sus síntomas y de su calidad de vida (los puntajes obtenidos por las pacientes después de la mamoplastía fueron mayores a los registrados antes del procedimiento).

Algunas limitaciones de nuestro trabajo son, entre otras, que pacientes y controles son de la región metropolitana, no incluyendo aquellas pacientes de otras regiones del país. No se incluyó una medida del volumen mamario, ya que no hay consenso en la literatura respecto a su valor diagnóstico. En estas pacientes es muy importante la proporción existente entre peso mamario y contextura, lo que depende de factores no estric- tamente relacionados al volumen mamario como medida aislada ${ }^{38}$.

En suma, nuestro estudio muestra que la traducción al español del cuestionario de síntomas relacionados a la mama (Breast Related Symptoms) es un instrumento válido y confiable para evaluar calidad de vida específica en pacientes chilenas portadoras de hipertrofia mamaria sintomática. La información obtenida en este estudio será de utilidad en la evaluación médica general de una paciente que reporta síntomas asociados al mayor tamaño de sus mamas, en la indicación de tratamiento y evaluación de sus resultados.

Agradecimientos: A los Drs. Annerleim Walton, Roberto González Lagos y José Ignacio Moretti

\section{Referencias}

1. Sanders C, Egger M, Donovan J, Tallon D, Franquel S. Reporting on quality of life in randomizae controlled trials: Bibliographic study. BMJ 1998; 317: 1191-4.

2. Testa M, Simonson D. Assessment of quality of life outcomes. N Engl J Med 1996; 334: 835-40.

3. Serón P, Riedermann G, Sanhueza A, Doussoulin A, Villarroel P. Validación del Cuestionario de la Enfermedad respiratoria crónica en pacientes chilenos con limitación crónica del flujo aéreo. Rev Med Chile 2003; 131: 1243 50.

4. Güell R, Casan P, Sangenís M, Morante F, Belda J, Guyatt G. Quality of life in patients with chronic respiratory disease: the spanish version of the Chronic Respiratory Questionnaire (CRQ). Eur Respir J 1998; 11: 55-60.

5. Burke C. Testing an asthma quality of life model. Journal of theory construction \& testing 2001; 5: 38-44.

6. Bowling A. Health-related quality of life: conceptual meaning, use and measurement. En Bowling A, Measuring health: a review of quality of life measurement scales. Philadelphia, USA: Editorial Open University Press; 1991.p. 1-19.

7. Urzúa A. Calidad de vida relacionada a la salud: elementos conceptuales. Rev Med Chile 2010; 138: 358-65.

8. Nanda U, Andresen E. Health related quality of life, a guide for the health professional. Evaluation \& the Health Professions 1998; 21: 179-215.

9. Guyat G, Feeny D, Patrick D. Measuring Health-related quality of life. Annals of Internal Medicine 1993; 18: 622-9.

10. Jones G. Breast reduction. En Mathes S, Editor Hentz V, Plastic Surgery. Philadelphia, USA: Editorial Elsevier; 
2006. p. 539-84.

11. Brown D, Young V. Reduction mammaplasty for macromastia. Aesthetic Plast. Surg 1993; 17: 211-23.

12. Spector J, Kart N. Reduction mammaplasty: a significant improvement at any size. Plast Recon Surg 2005; 15: 845 50.

13. Centineo M, Zarza L, Razeto. Alteraciones de la columna vertebral en las hipertrofias mamarias. Cir Plas Iberolatinoam 1989; 15: 91.

14. Faria F, Guthrie E, Bradbury E. Psychosocial outcome and patients satisfaction following breast reduction surgery. Br J Plast Surg 1999; 52: 448-52.

15. Rioja L, Pedreño F, Deza P, Benítez J, Cabrera E, Redondo A, et al. Hipertrofia mamaria, ¿una deformidad estética? Cuándo debe sufragarse por la seguridad social. Cir Plas Iberolatinoam 2006; 32: 99-106.

16. Thoma A, Sprange S, Veltri K, Duku E, Furlong W. A prospective study of patients undergoing breast reduction surgery: health - related quality of life and clinical outcomes. Plast Reconstr Surg 2007; 120 (1): 13-26.

17. Kerrigan C, Collins E, Striplin D, Kim H, Wilkins E, et al. The health burden of breast hypertrophy. Plast Reconstr Surg. 2001; 108 (6): 1591-9.

18. Collins E, Kerrigan G, Lowery J. The effectiveness of surgical and nonsurgical interventions in relieving the symptoms of macromastia. Plast Reconstr Surg 2002; 109 (5): 1556-66.

19. Torrance G, Feeny D, Furlong W. Multiattribute utility function for a comprehensive health status classification system: Health Utilities Index Mark 2.1996. Med Care 1996; 34: 702-22.

20. Horsman J, Furlong B, Feeny D. The Health Utilities Index (HUI): Concepts, measurement properties, and applications. Health Qual Life Outcomes 2003; 16: 5467.

21. Martínez M. Validez y confiabilidad en la metodología cualitativa. Paradìgma 2006; 27 (2): 7-33.

22. Streiner D. Checklist for evaluating the usefulness of rating scales. Can J Psychiatry 1993; 38: 140-8.

23. Souza R, González E, Peña S, Güemes A, García-Campayo J. Aspectos psicosomáticos de la hipertrofia mamaria. Psiquis 2003; 24 (4): 31-6.

24. O’Blenes C, Delbridge C, Miller B, Pantelis A, Morris S. Prospective study of outcomes after reduction mammaplasty: long-term follow-up. Plast Reconstr Surg 2006; 117 (2): 351-58.
25. Aguilera B, Escobar P, Ruiz de Viñaspre P, Uribe A, Celis M, Gavilán P, et al. Reducción mamaria con técnica de pedículo inferior. Evaluación de la satisfacción de las pacientes de la Unidad de Mastología del Hospital Santiago Oriente Dr. Luis Tisné Brousse. Rev Obstet Ginecol 2006; 1 (2): 100-4.

26. Millar B, Morris S, Sigurdson L, Bendor-Samuel R, Brennan M, Davis G, et al. Prospective study of outcomes after reduction mammaplasty. Plast Reconstr Surg 2005; 115 (4): 1025-31.

27. Chao J, Memmel H, Redding J, Egan L, Odom L, Casas L. Reduction mammaplasty is a functional operation, improving quality of life in symptomatic women: a prospective, single-center breast reduction outcome study. Plast Reconstr Surg 2002; 110 (7): 1644-52.

28. Kerrigan C. Prospective study of outcomes after reduction mammaplasty (discussion). Plast Reconstr Surg 2005; 115: 1032-3.

29. Espinosa A, De la Torre J. Lejour Breast Reduction. Updated: Sep 30, 2009. Disponible en: emedicine.medscape. com/article/1274770-overview

30. Hall-Findlay E. Breast Reduction. En: Mc Carthy J, Galiano R, Boutros S. Editores, Current Therapy in Plastic Surgery. Philadelphia, USA: Saunders Elsevier 2006. p. 403-10.

31. Jacubovsky D. Mamoplastía Reductiva. En: Calderón W, Cirugía Plástica. Santiago, Chile: Sociedad de Cirujanos de Chile; 2001. P. 599-618.

32. Schnur P, Schnur D, Petty P, Hanson T, Weaver A. Reduction mammaplasty: An outcome study. Plast Reconstr Surg 1997; 100: 875-83.

33. Kerrigan C, Sampalis J. Reduction mammaplasty: Impact on patient outcome. Can J Plast Surg 1997; 5: 39.

34. Barret R. An evidence-bades approach to reduction mammaplasty. Plast Reconstr Surg 2010; 126: 2171-6.

35. Kerrigan C, Collins E, Kim H. Reduction mammaplasty: Defining medical necessity. Med Decis Making 2002; 22: 208-17.

36. Taboada S, Brea G, González A, Couto G. Grado de satisfacción tras cirugía de hipertrofia mamaria severa. Cir Plast Iberolatinoam 2010; 36: 13-8.

37. Gúemes S, Souza D, Salinas P, Torcal A, Burdío P, Beltrán J, et al. Mamoplastía de Reducción. Indicaciones y consideraciones técnicas. Cir Esp 2000; 68: 30-4.

38. Adams W. Reduction Mammaplasty and Mastopexy. SRPS 2002; 9 (29): 1-48. 